\title{
Entschließung der DSK des Bundes und der Länder vom 24. Januar 2017
}

Unter dem Vorsitz der Landesbeauftragten für den Datenschutz Niedersachsen hat die Konferenz der unabhängigen Datenschutzbehörden des Bundes und der Länder am 24. Januar 2017 in Hannover getagt.

\section{Entschließung "Novellierung des Personalausweisgesetzes - Änderungen müssen bürger- und datenschutzfreundlich realisiert werden!"}

Die Bundesregierung plant grundlegende Änderungen des Personalausweisrechts. Nach dem vom Bundeskabinett beschlossenen Gesetzentwurf (BR-Drs. 787/16) werden das Recht auf informationelle Selbstbestimmung der Bürgerinnen und Bürger übergangen und Datenschutz sichernde Standards unterlaufen. Die Konferenz der unabhängigen Datenschutzbehörden des Bundes und der Länder fordert daher insbesondere folgende datenschutzrechtliche Anforderungen zu berücksichtigen:

- Die obligatorische Aktivierung der eID-Funktion ist dann hinnehmbar, wenn dauerhaft sichergestellt ist, dass daraus keine verpflichtende Nutzung der eIDFunktion des Personalausweises resultiert. Die Entscheidung über die Nutzung der eIDFunktion muss allein bei den Bürgerinnen und Bürgern liegen. Deren Selbstbestimmungsrecht muss gewahrt bleiben.

- An der bisherigen Verpflichtung der Ausweisbehörden, Bürgerinnen und Bürger über die eID-Funktion des Personalausweises schriftlich zu unterrichten, sollte festgehalten werden. Nur durch eine bundesweit einheitliche Vorgabe zu einer solchen Information wird sichergestellt, dass alle Bürgerinnen und Bürger in hinreichend verständlicher Form aufgeklärt werden.

- Vor einer Datenübermittlung aus dem Personalausweis müssen die Bürgerinnen und Bürger Kenntnis über den Zweck der Übermittlung erhalten; zur Wahrnehmung des Rechts auf informationelle Selbstbestimmung müssen die Betroffenen stets - wie bislang - nachvollziehen können, in welchem konkreten Kontext ihre Identitätsdaten übermittelt werden. Zudem sollte die bisherige Möglichkeit, die Übermittlung einzelner Datenkategorien auszuschließen, beibehalten werden.

- Die Einführung von organisationsbezogenen Berechtigungszertifikaten bei Diensteanbietern wird abgelehnt. Um sicherzustellen, dass Diensteanbieter nur die für den jeweiligen Ge- schäftsprozess erforderlichen Angaben übermittelt bekommen, sollte an der aktuellen Rechtslage festgehalten werden, nach der der antragstellende Diensteanbieter die Erforderlichkeit der aus der eIDFunktion des Personalausweises zu übermittelnden Angaben nachweisen muss und an den jeweils festgelegten Zweck gebunden ist.

- Berechtigungszertifikate dürfen nur an Diensteanbieter erteilt werden, die Datenschutz und Datensicherheit gewährleisten. Daher sollten antragstellende Diensteanbieter nach wie vor durch eine Selbstverpflichtung die Erfüllung dieser Anforderungen schriftlich bestätigen und nachweisen müssen.

- Die maßgeblichen Regelungen für die mit der Anlegung und Nutzung von Servicekonten einhergehende Erhebung und Verarbeitung von Identitätsdaten aus dem Personalausweis sowie die sicherheitstechnischen Rahmenbedingungen sollten im Personalausweisgesetz getroffen werden.

- Die Voraussetzungen für die Erstellung und Weitergabe von Personalausweisablichtungen sollten gesetzlich konkreter normiert werden. Insbesondere das Prinzip der Erforderlichkeit ist durch eine verpflichtende Prüfung der Notwendigkeit der Anfertigung einer Ablichtung sowie durch eine Postitivliste von Erlaubnisgründen zu stärken. Die Einwilligung der Betroffenen als alleinige Voraussetzung birgt die Gefahr, dass in der Praxis Ablichtungen angefertigt werden, obwohl sie nicht erforderlich sind. Zudem dürfte fraglich sein, ob betroffene Personen in eine solche Maßnahme stets informiert und freiwillig einwilligen können.

- Die zum 1. Mai 2021 vorgesehene Einführung eines nahezu voraussetzungslosen Abrufs des Lichtbildes im automatisierten Verfahren durch die Polizeibehörden des Bundes und der Länder sowie die Verfassungsschutzbehörden und Nachrichtendienste wird abgelehnt. Bisher dürfen zur Verfolgung von Straftaten und Verkehrsordnungswidrigkeiten insbesondere die Polizei- und Ordnungsbehörden Lichtbilder automatisiert abrufen, wenn die Personalausweisbehörde nicht erreichbar ist und ein weiteres Abwarten den Ermittlungszweck gefährdet. Diese gesetzlichen Einschränkungen für das Abrufverfahren sollen nun entfallen. Zudem sollen alle Nachrichtendienste künftig voraussetzungslos Lichtbilddaten abrufen können. Die bisherige Rechtslage ist völlig ausreichend. 